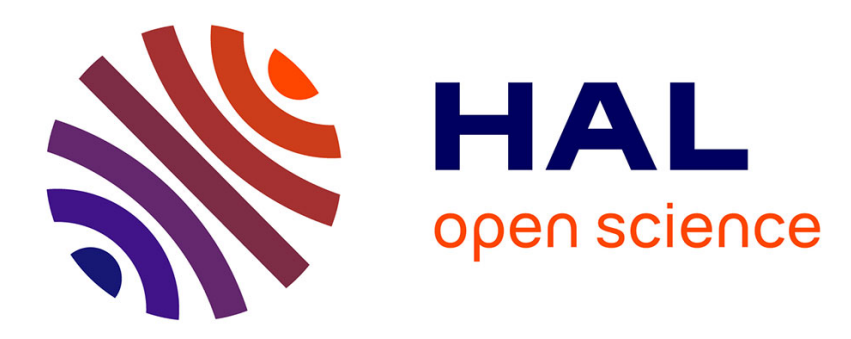

\title{
Effect of next-nearest-neighbour hopping on BoseEinstein condensation in optical lattices
} T A Zaleski, T K Kope

\section{To cite this version:}

T A Zaleski, T K Kope. Effect of next-nearest-neighbour hopping on BoseEinstein condensation in optical lattices. Journal of Physics B: Atomic, Molecular and Optical Physics, 2010, 43 (8), pp.85303. 10.1088/0953-4075/43/8/085303 . hal-00630009

\section{HAL Id: hal-00630009 \\ https://hal.science/hal-00630009}

Submitted on 7 Oct 2011

HAL is a multi-disciplinary open access archive for the deposit and dissemination of scientific research documents, whether they are published or not. The documents may come from teaching and research institutions in France or abroad, or from public or private research centers.
L'archive ouverte pluridisciplinaire HAL, est destinée au dépôt et à la diffusion de documents scientifiques de niveau recherche, publiés ou non, émanant des établissements d'enseignement et de recherche français ou étrangers, des laboratoires publics ou privés. 


\title{
Effect of next-nearest-neighbour hopping on Bose-Einstein condensation in optical lattices
}

\author{
T. A. Zaleski and T. K. Kopeć \\ Institute of Low Temperature and Structure Research, \\ Polish Academy of Sciences, POB 1410, 50-950 Wrocław 2, Poland \\ E-mail: T.Zaleski@int.pan.wroc.pl
}

\begin{abstract}
We study the influence of the various hopping elements on the ground state properties of the bosons in the $2 \mathrm{D}$ optical square lattice. Using the quantum rotor approach for the Bose-Hubbard model with nearest $t$ and next nearest-neighbours $t^{\prime}$ hopping, we quantify to what extent changing the ratio $t^{\prime} / t$ affects the superfluid to Mott-insulator transition.
\end{abstract}

PACS numbers: $67.85 . \mathrm{Hj}, 67.85 . \mathrm{Bc}, 03.75 . \mathrm{Hh}$

\section{Introduction}

Optical lattices offer remarkably clean access to a particular Hamiltonian and, thereby, serve as a model system for testing fundamental theoretical concepts and providing an exemplar of quantum many-body effects [1]. It is well known that the ground state of a system of repulsively interacting bosons in a periodic potential can be either in a superfluid (SF) state or in a Mott-insulating (MI) state, characterized by integer boson densities [2]. A periodic lattice potential with tightly confining potential wells can be created by realising a dipole trap with superimposed counter propagating laser beams [3]. These beams interfere and the interference pattern results in a periodic potential landscape. For example, to form a two-dimensional lattice potential two standing waves can be superimposed orthogonal to each other. The strength of the tunnel coupling is characterized by the tunnel matrix element

$$
t_{i j}=-\int d \mathbf{r} w_{0}\left(\mathbf{r}-\mathbf{r}_{i}\right) \hat{H}_{0} w_{0}\left(\mathbf{r}-\mathbf{r}_{j}\right)
$$

where $\hat{H}_{0}$ is the Hamiltonian of the system, which includes the periodic lattice potential. This element can be calculated by considering two Wannier functions of neighbouring lattice sites $\mathbf{r}_{i}$ and $\mathbf{r}_{j}$ :

$$
w_{n}\left(\mathbf{r}-\mathbf{R}_{i}\right) \equiv \frac{1}{N} \sum_{\mathbf{q}} e^{-i \mathbf{q} \cdot \mathbf{R}_{i}} \psi_{n \mathbf{q}}(\mathbf{r}),
$$

where $\psi_{n \mathbf{q}}(\mathbf{r})=u_{n \mathbf{q}}(\mathbf{r}) e^{i \mathbf{q} \cdot \mathbf{r}}$, with $u_{n \mathbf{q}}(\mathbf{r})=u_{n \mathbf{q}}\left(\mathbf{r}+\mathbf{a}_{i}\right)$ being a periodic function, $n$ is the band index and $\mathbf{a}_{i}$ is a lattice vector. If a sample of sufficiently cold bosonic atoms 
is loaded into a deep enough lattice (e.g. from a Bose-Einstein condensate), then only states of the lowest band will be populated. Since, the Wannier state is well localized, $t_{i j}$ rapidly decreases with increasing relative separation $\left|\mathbf{r}_{i}-\mathbf{r}_{j}\right|$. Usually, the second nearest neighbours have tunnelling rates that are 2-4 orders of magnitude smaller than those of the nearest neighbours and the tight-binding can be expected to provide an excellent description of the system in this regime. This represents the so-called tight binding limit, where a state at any given site only couples to a few neighbouring sites. However, it turns out that the Wannier state of the two-dimensional (2D) square lattice can be weakly localized [4] (see, Fig. 1) depending on the intensity of the lattice. We note that, even in 1D one can have regimes where next nearest-neighbour coupling is or is not relevant [5]. Therefore, it appears reasonable to quantify the influence of the next nearest-neighbour sites on the ground state properties of the bosons in the 2D optical lattice. Furthermore, due to the separability of the potential for the 2D square lattice, the tunnelling to sites lying on diagonals is identically equal to zero. As a consequence, the tunnelling matrix arrangement including nearest and next to nearest neighbours hopping is restricted to the lattice axes, as shown in Fig. 1.

\section{Model}

We start from a second quantized purely bosonic Hubbard Hamiltonian:

$$
\begin{aligned}
\mathcal{H}= & -t \sum_{\left\langle\mathbf{r}, \mathbf{r}^{\prime}\right\rangle}\left[a^{\dagger}(\mathbf{r}) a\left(\mathbf{r}^{\prime}\right)+a^{\dagger}\left(\mathbf{r}^{\prime}\right) a(\mathbf{r})\right] \\
& -t^{\prime} \sum_{\left\langle\left\langle\mathbf{r}, \mathbf{r}^{\prime}\right\rangle\right\rangle}\left[a^{\dagger}(\mathbf{r}) a\left(\mathbf{r}^{\prime}\right)+a^{\dagger}\left(\mathbf{r}^{\prime}\right) a(\mathbf{r})\right] \\
& +\frac{U}{2} \sum_{\mathbf{r}} n^{2}(\mathbf{r})-\bar{\mu} \sum_{\mathbf{r}} n(\mathbf{r}),
\end{aligned}
$$

where, $a^{\dagger}(\mathbf{r})$ and $a\left(\mathbf{r}^{\prime}\right)$ are bosonic creation and annihilation operators defined on sites $\mathbf{r}$ and $\mathbf{r}^{\prime}$ of a regular square lattice. The summations are performed over the nearest $\langle\ldots\rangle$ and the next-nearest neighbours $\langle\langle\ldots\rangle\rangle$, respectively with hoppings $t$ and $t^{\prime}$ (see, Fig. 1). A dispersion relation of the lattice is:

$$
\varepsilon(\mathbf{k})=2 t\left(\cos k_{x}+\cos k_{y}\right)+2 t^{\prime}\left(\cos 2 k_{x}+\cos 2 k_{y}\right) .
$$

Furthermore, $U$ is the on-site repulsion energy, $n(\mathbf{r})=a^{\dagger}(\mathbf{r}) a(\mathbf{r})$ represents the boson number operator, while $\bar{\mu}=\mu+\frac{U}{2}$, with $\mu$ being a chemical potential controlling the average number of bosons. The Bose-Hubbard model in Eq. (3) has been studied intensively for a number of years $[6,7]$ and recently has been applied to bosons in optical lattices $[8,9]$. A number of approaches has been applied to investigate the physics of the model like mean-field theories [10], strong-coupling expansion [11, 12], coarse graining [13], methods based on a systematic strong-coupling approach, of the Bose-Hubbard model, but going beyond mean field model $[15,16]$, or numerical once, e.g. quantum Monte Carlo [17, 18, 19]. 


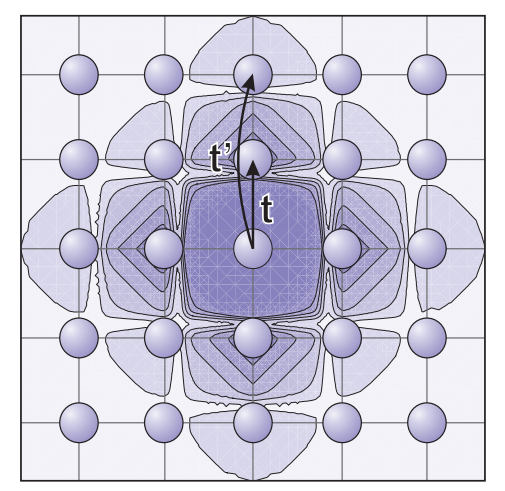

Figure 1. (Colour online) Two-dimensional square lattice with the nearest neighbours $t$ and the next-nearest neighbours $t^{\prime}$ hopping. Shaded areas denote Wannier state density distribution based on data from Ref. [4].

\section{Quantum rotor representation}

In the following, we switch from the particle-number representation to the conjugate phase representation of the bosonic degrees of freedom which constitutes an effective bosonic description of the strongly correlated system [16]. To this aim the secondquantized Hamiltonian of the model is translated to the phase representation with the help of the topologically constrained path integral formalism. A similar method that is based on quantum rotor formulation was recently employed by one of us in the fermionic Hubbard model [20]. The best merit of this approach is that it is capable of handling spatial and quantum fluctuation effects properly and complying with Mermin-Wagner theorem [21]. It also gives the results that are in a very good agreement with Quantum Monte Carlo method (see, Fig. 2 in Ref. [16]).

The statistical sum can be written in a path integral form with use of customary complex fields, $a(\mathbf{r} \tau)$ depending on the "imaginary time" $0 \leq \tau \leq \beta \equiv 1 / k_{B} T$, (with $T$ being the temperature) that satisfy the periodic condition $a(\mathbf{r} \tau)=a(\mathbf{r} \tau+\beta)$ :

$$
\mathcal{Z}=\int[\mathcal{D} \bar{a} \mathcal{D} a] e^{-\mathcal{S}[\bar{a}, a]}
$$

where the action $\mathcal{S}$ is equal to:

$$
\mathcal{S}[\bar{a}, a]=\sum_{\mathbf{r}} \int_{0}^{\beta} d \tau\left[\bar{a}(\mathbf{r} \tau) \frac{\partial}{\partial \tau} a(\mathbf{r} \tau)+\mathcal{H}(\tau)\right] .
$$

Next, we performing a local gauge transformation to new bosonic variables:

$$
\left[\begin{array}{c}
a(\mathbf{r} \tau) \\
\bar{a}(\mathbf{r} \tau)
\end{array}\right]=\left[\begin{array}{cc}
e^{i \phi(\mathbf{r} \tau)} & 0 \\
0 & e^{-i \phi(\mathbf{r} \tau)}
\end{array}\right]\left[\begin{array}{c}
b(\mathbf{r} \tau) \\
\bar{b}(\mathbf{r} \tau)
\end{array}\right] .
$$

The U(1) group governing the phase field $\phi(\mathbf{r} \tau)$ is compact, i.e. $\phi(\mathbf{r} \tau)$ has the topology of a circle $S_{1}$. The chief merit of the transformation in Eq. (7) is that we have managed to cast the strongly correlated bosonic problem into a system of weakly interacting 
bosons, submerged in the bath of strongly fluctuating $U(1)$ gauge potentials on the high energy scale set by $U$. In the phase-only representation, the statistical sum reads:

$$
Z=\int[\mathcal{D} \phi] e^{-\mathcal{S}_{p h}[\phi]}
$$

with the action:

$$
\begin{aligned}
\mathcal{S}_{p h}[\phi]= & \int_{0}^{\beta} d \tau\left\{\sum_{\mathbf{r}}\left[\frac{\dot{\phi}^{2}(\mathbf{r} \tau)}{2 U}+i \frac{\bar{\mu}}{U} \dot{\phi}(\mathbf{r} \tau)\right]\right. \\
& -J \sum_{\left\langle\mathbf{r}, \mathbf{r}^{\prime}\right\rangle} \cos \left[\phi(\mathbf{r} \tau)-\phi\left(\mathbf{r}^{\prime} \tau\right)\right] \\
& -J^{\prime} \sum_{\left\langle\left\langle\mathbf{r}, \mathbf{r}^{\prime}\right\rangle\right\rangle} \cos \left[\phi(\mathbf{r} \tau)-\phi\left(\mathbf{r}^{\prime} \tau\right)\right]
\end{aligned}
$$

where $\dot{\phi}(\mathbf{r} \tau)=\partial \phi(\mathbf{r} \tau) / \partial \tau$. We parametrize the boson fields as follows

$$
b(\mathbf{r} \tau)=b_{0}+b^{\prime}(\mathbf{r} \tau)
$$

and restrict our calculations to the fixed amplitude $b_{0}$ thus, dropping the amplitude fluctuation term $b^{\prime}(\mathbf{r} \tau)$. This is justified by the observation that in the strong coupling limit $t / U \ll 1$ the physics of the problem under study is dominated by the behaviour in the $\mathrm{U}(1)$ sector. As a consequence, $J=\left|b_{0}\right|^{2} t, J^{\prime}=\left|b_{0}\right|^{2} t^{\prime}$ and the integration over phase variables is restricted over the compact $\mathrm{U}(1)$ group manifold [22]. In performing this integration one should take phase configurations that satisfy the boundary condition

$$
\phi(\mathbf{r} \beta)-\phi(\mathbf{r} 0)=2 \pi m(\mathbf{r})
$$

and $m(\mathbf{r})=0, \pm 1, \pm 2, \ldots$. By the definition, for the superfluid transition the order parameter reads:

$$
\boldsymbol{\Psi}_{B}=\langle a(\mathbf{r} \tau)\rangle=\langle b(\mathbf{r} \tau)\rangle \psi_{B} .
$$

Its non-vanishing signals a bosonic condensation (we identify it as superfluid state). Note that a nonzero value of the amplitude $\langle b(\mathbf{r} \tau)\rangle$ is not sufficient for superfluidity. To achieve this, also the phase variables, must become stiff and coherent, which implies that $\psi_{B}$ defined as:

$$
\psi_{B}=\left\langle e^{i \phi(\mathbf{r} \tau)}\right\rangle_{p h}
$$

is non-vanishing. The averages appearing in Eqs. (12) and (13) are as follows:

$$
\langle\ldots\rangle=\frac{\int[\mathcal{D} \bar{\xi} \mathcal{D} \xi] \ldots e^{-\mathcal{S}[\bar{\xi}, \xi]}}{\int[\mathcal{D} \bar{\xi} \mathcal{D} \xi] e^{-\mathcal{S}[\bar{\xi}, \xi]}}
$$

where $\xi$ denotes $a$ or $b$ fields, respectively,

$$
\langle\ldots\rangle_{p h}=\frac{\int[\mathcal{D} \phi] \ldots e^{-\mathcal{S}_{p h}[\phi]}}{\int[\mathcal{D} \phi] e^{-\mathcal{S}_{p h}[\phi]}}
$$

and the action $\mathcal{S}_{p h}[\phi]$ given in Eq. (9). 
The value of the boson amplitude $b_{0}$ can be obtained from saddle-point condition:

$$
\left.\frac{\partial f[\bar{b}, b]}{\partial b}\right|_{b=b_{0}}=\left.\frac{\partial f[\bar{b}, b]}{\partial \bar{b}}\right|_{\bar{b}=\bar{b}_{0}}=0
$$

with the free energy per lattice site $f=-\frac{1}{\beta N} \ln \int \mathcal{D} \bar{b} \mathcal{D} b e^{-\mathcal{S}[\bar{b}, b]}$. Using the parametrization in Eq. (10) we obtain the action :

$$
\mathcal{S}\left[\bar{b}_{0}, b_{0}\right]=N \beta\left[-\left(z t+z^{\prime} t^{\prime}\right)\left|b_{0}\right|^{2}-\bar{\mu}\left|b_{0}\right|^{2}+\frac{U}{2}\left|b_{0}\right|^{4}\right] .
$$

So that, the amplitude of the bosonic field reads:

$$
\left|b_{0}\right|^{2}=2\left(\frac{z t+z^{\prime} t^{\prime}}{U}+\frac{\bar{\mu}}{U}\right)
$$

where $z$ and $z^{\prime}$ are number of nearest neighbours and next-nearest neighbours, respectively, for a given lattice (in our case, $z=z^{\prime}=4$ ).

The definition in Eq. (13) of the order parameter suggests that the description in terms of the unimodular complex field $\psi(\mathbf{r} \tau)=e^{i \phi(\mathbf{r} \tau)}\left(|\psi(\mathbf{r} \tau)|^{2}=1\right)$ would be more desirable than working a with trigonometric action involving the phase variable $\phi(\mathbf{r} \tau)$. Furthermore, we substitute the rigid unimodular constraint $|\psi(\mathbf{r} \tau)|^{2}=1$ for each site $\mathbf{r}$ by the weaker spherical closure relation, which maps the problem onto the soluble quantum spherical (QS) model [16]. The convenient way to enforce the spherical constraint is the functional analog of the $\delta$-function representation $\delta(x)=$ $\int_{-\infty}^{+\infty}(d \lambda / 2 \pi i) e^{\lambda x}$, which introduces the Lagrange multiplier $\lambda$. The evaluation of the effective action in terms of the $\psi(\mathbf{r} \tau)$ fields may be organized using the loop method [23]. To the second order in $\psi(\mathbf{r} \tau)$ we obtain the partition function of the effective QS model:

$$
\mathcal{Z}=\frac{1}{2 \pi i} \int_{-i \infty}^{+i \infty} d \lambda \int[\mathcal{D} \psi \mathcal{D} \bar{\psi}] e^{-\mathcal{S}[\psi, \bar{\psi}]}
$$

with the Fourier transformed action:

$$
\mathcal{S}\left[\psi, \bar{\psi}=-\frac{1}{N \beta} \sum_{\mathbf{k} \ell}\left[J(\mathbf{k})-\gamma^{-1}\left(\omega_{\ell}\right)-\lambda\right] \psi\left(\mathbf{k} \omega_{\ell}\right) \bar{\psi}\left(\mathbf{k} \omega_{\ell}\right),\right.
$$

where the Bose-Matsubara frequencies are $\omega_{\ell}=2 \pi \ell / \beta$ with $\ell=0, \pm 1, \pm 2, \ldots$ Here, the dispersion for the nearest and the next-nearest neighbours phase stiffness parameters is given by

$$
J(\mathbf{k})=2 J\left(\cos k_{x}+\cos k_{y}\right)+2 J^{\prime}\left(\cos 2 k_{x}+\cos 2 k_{y}\right),
$$

where $J=t\left|b_{0}\right|^{2}$ and $J^{\prime}=t^{\prime}\left|b_{0}\right|^{2}$ with $b_{0}$ given in Eq. (18). Furthermore, $\gamma\left(\omega_{\ell}\right)$ is Fourier transformed phase correlation function:

$$
\gamma\left(\mathbf{r} \tau ; \mathbf{r}^{\prime} \tau^{\prime}\right)=\left\langle e^{-i\left[\phi(\mathbf{r} \tau)-\phi\left(\mathbf{r}^{\prime} \tau^{\prime}\right)\right]}\right\rangle_{p h}
$$

which explicit form is given in Ref. [16]. 


\section{Results}

Considering the problem at the mean-field level, it is obvious from Eq. (18) that the effect of $t^{\prime}$ is a simple correction to the direct hopping $t$. However, as already mentioned in a strongly correlated case $t \ll U$ the actual superfluid order is determined by the phase coherence among bosons rather than the non-vanishing amplitude of the Bose field. Therefore, going beyond the mean-field result using quantum rotor method it is not a priori clear what the influence of $t^{\prime}$ will be, especially for a two-dimensional system, where phase fluctuations are significant. As usual, in the quantum rotor model calculations the critical boundary is determined from the condition of vanishing of the order parameter susceptibility $G^{-1}\left(\mathbf{k}=0, \omega_{\ell}=0\right)=0$, which defines the value of the Lagrange multiplier $\lambda_{0}$ at criticallity. Here,

$$
G^{-1}\left(\mathbf{k} \omega_{\ell}\right)=\lambda_{0}-J(\mathbf{k})+\gamma^{-1}\left(\omega_{\ell}\right) .
$$

The "equation of state" with the order parameter $\left|\psi_{B}\right|^{2}$ reads:

$$
1-\left|\psi_{B}\right|^{2}=\frac{1}{\beta N} \sum_{\mathbf{k} \ell} G\left(\mathbf{k} \omega_{\ell}\right) .
$$

Because in two-dimensions a long-range order is expected only in the ground state we have to specialize in the zero temperature limit $\beta \rightarrow \infty$ in Eq. (24). We obtain:

$$
1-\left|\psi_{B}\right|^{2}=\frac{1}{N} \int_{-\infty}^{+\infty} \frac{d \varepsilon \rho(\varepsilon)}{2 \sqrt{\frac{\lambda_{0}-t\left|b_{0}\right|^{2} \varepsilon}{U}+\frac{1}{4}}},
$$

where the critical value of the Lagrange multiplier is:

$$
\lambda_{0}=J(0)+\gamma^{-1}(0)=\left|b_{0}\right|^{2} \varepsilon(\mathbf{0})-\frac{U}{4}+v^{2}\left(\frac{\mu}{U}\right)
$$

with a periodic function $v[x]=x-[x]-1 / 2$ resulting from a boundary condition in Eq. (11), where $[x]$ is the floor function giving the greatest integer less than or equal to $x$. In order to perform summation over wave vectors in equation of state in Eq. (24), it is desirable to introduce a density of states of the $2 \mathrm{D}$ lattice with the nearest $t$ and next-nearest $t^{\prime}$ neighbours hopping:

$$
\rho(\epsilon)=\frac{1}{N} \sum_{\mathbf{k}} \delta\left[\epsilon-\frac{\varepsilon(\mathbf{k})}{t}\right]
$$

which can be conveniently written as a convolution involving one dimensional densities of states:

$$
\rho(\epsilon)=\int_{-\infty}^{+\infty} d x \Lambda(x) \Lambda(\epsilon-x)
$$

where

$$
\Lambda(x)=\Theta[\zeta(x)] \frac{\rho_{1 D}\left[\frac{1-\sqrt{\zeta(x)}}{4 r}\right]+\rho_{1 D}\left[\frac{1+\sqrt{\zeta(x)}}{4 r}\right]}{\sqrt{\zeta(x)}}
$$




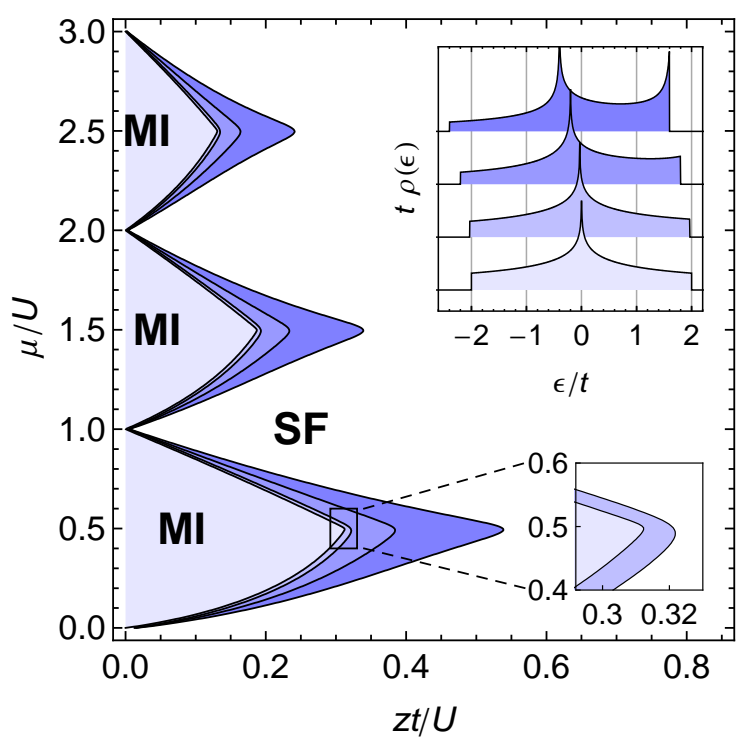

Figure 2. (Colour online) Zero-temperature phase diagram of the system as a function of hopping and chemical potential. Lobes for various values of the nearest neighbours $t^{\prime}$ to the next-nearest neighbours hopping $t$ ratios (from the lightest to the darkest shading: $\left.t^{\prime} / t=0,-0.016,-0.1,-0.2\right)$. MI denotes a Mott Insulator phase, while SF is a superfluid region. The lower inset shows details of the $t^{\prime}=0$ and $t^{\prime} / t=-0.016$ lobes. The upper inset shows the lattice density of states calculated from the dispersion in Eq. (4) and Eq. (28) for the respective ratios of $t^{\prime} / t$.

with $\eta=t^{\prime} / t$, furthermore $\zeta(x)=1+8 r(x+r), \Theta(x)$ is a step function and the one-dimensional density of states is given by $\rho_{1 D}(x)=(1 / \pi) \Theta(1-|x|) / \sqrt{1-x^{2}}$. For $t^{\prime} / t=0$ we recover the usual density of state for the $2 \mathrm{D}$ lattice with the logarithmic singularity at $\epsilon=0$. For $t^{\prime}>0$, the density of states given by Eq. (28) is rather uncommon e.g. in solid state physics applications, because usually the term next nearestneighbours hopping refers to the interactions along diagonals of the square lattice, which are vanishing in the present case. In the MI phase, bosons are incompressible $\partial n_{B} / \partial \mu=0$ and localized, which means that the total energy is minimized when each site is filled with the same number of atoms. Increasing fluctuations in the phase system reduces fluctuations in the boson number on each site according to the Heisenberg uncertainty relation $\Delta n_{B} \Delta \phi \geq 1 / 2$. By crossing the boundary line, bosons can move from one lattice site to the next. The order parameter $\boldsymbol{\Psi}_{B}$ has a non-vanishing value, and the system exhibits a long-range phase coherence. This is opposite to the case of the Mott insulator, where phase coherence is lost. In Fig. 2 we plotted zero-temperature phase diagram as a function of normalized hopping $t / U$ vs. normalized chemical potential $\mu / U$ for various values of $t^{\prime} / t$ hopping parameter. The phase boundary is periodic with the chemical potential $\mu / U$ and the filling is fixed to the integer values within the lobes. For square lattice, numerical investigation of the band structure and explicit calculation of the Wannier states give the negative sign of $t^{\prime} / t$ ratio with value of $t^{\prime} / t=-0.016\left(t^{\prime}\right.$ being $1.6 \%$ of $t$, see, Table 1 in Ref. [4]. In our calculations, it leads to an increase of the size of the lobes by approximately $3 \%$ with respect to the 
pure $2 \mathrm{D}$ case with the nearest neighbours interactions only. Also, the shape of the lobes changes with increasing $t^{\prime}$ hopping. In general, the inclusion of the next-nearest neighbours suppresses the superfluid state (see, Fig. 2). From Ref. [4] it follows that for other type of lattices like hexagonal or rectangular the respective absolute value of $t^{\prime} / t$ is even smaller than in the present case, which suggest that the prospective effects on the phase diagram will be even less pronounced.

\section{Conclusions}

In this work, we have investigated two-dimensional lattices with nearest and next-nearest neighbours hopping that may be used in experiments with Bose condensation of cold atoms. We have shown that lattices with next-nearest neighbours hopping could affect the ground state interaction - chemical potential phase diagram of the system, which consist of Mott insulating lobes and superfluid region by suppressing the superfluid state. In a view towards promising future developments, it would be interesting to consider experimental realization of the presented scenario by means of two-dimensional optical lattices with changing lattice potential depth.

\section{Acknowledgments}

Present work is supported from scientific financial resources in the years 2009-2012 as a research grant.

\section{References}

[1] I. Bloch, Nat. Phys. 1, 23 (2005).

[2] M. P. A. Fisher, P. B. Weichman, G. Grinstein, and D. S. Fisher, Phys. Rev. B 40, 546 (1989).

[3] O. Morsch, M. Oberthaler, Rev. Mod. Phys. 78, 179 (2006).

[4] P. B. Blakie, and C. W. Clark, J. Phys. B: At. Mol. Opt. Phys. 37, 1391 (2004).

[5] D. J. Boers, B. Goedeke, D. Hinrichs, and M. Holthaus, Phys. Rev. A 75, 063404 (2007).

[6] M. P. A. Fisher, P. B. Weichman, G. Grinstein, and D. S. Fisher, Phys. Rev. B 40, 546 (1989).

[7] S. Sachdev, Quantum Phase Transitions (Cambridge University Press, Cambridge, 1999).

[8] D. Jaksch, C. Bruder, J. I. Cirac, C. W. Gardiner, and P. Zoller, Phys. Rev. Lett. 81, 3108 (1998).

[9] M. Greiner, O. Mandel, T. Esslinger, T. W. Hansch, and I. Bloch, Nature (London) 415, 39 (2002).

[10] K. Sheshadri, H. R. Krishnamurthy, R. Pandit, and T. V. Ramakrishnan, Europhys. Lett. 22, 257 (1993).

[11] J. K. Freericks and H. Monien, Phys. Rev. B 53, 2691 (1996).

[12] N. Elstner and H. Monien, Phys. Rev. B 59, 12184 (1999).

[13] A. P. Kampf and G. T. Zimanyi, Phys. Rev. B 47, 279 (1993).

[14] W. Krauth, M. Caffarel, and J.-P. Bouchaud, Phys. Rev. B 45, 3137 (1992).

[15] K. Sengupta and N. Dupuis, Phys. Rev. A 71, 033629 (2005).

[16] T. P. Polak, and T. K. Kopeć, Phys. Rev. B 76, 094503 (2007).

[17] N. V. Prokofev, B. V. Svistunov, and I. S. Tupitsyn, Phys. Lett. A 238, 253 (1998); JETP 87, 310 (1998).

[18] F. Alet, S. Wessel, and M. Troyer, Phys. Rev. E 71, 036706 (2005).

[19] B. Capogrosso-Sansone, N. V. Prokofev, and B. V. Svistunov, Phys. Rev. B 75, 134302 (2007). 
[20] T. K. Kopeć, Phys. Rev. B 70, 054518 (2004).

[21] N. D. Mermin and H. Wagner, Phys. Rev. Lett. 17, 1133 (1966).

[22] L. S. Schulman, Techniques and Applications of Path Integration (Wiley, New York, 1981).

[23] T. K. Kopeć and J. V. José, Phys. Rev. B 60, 7473 (1999). 